\title{
Rapid near-infrared fluorescence excitation-emission matrix spectroscopy for multifluorophore characterization using an acousto-optic tunable filter technique
}

\author{
Hao Li \\ Wei Zheng \\ Zhiwei Huang \\ National University of Singapore \\ Faculty of Engineering \\ Department of Bioengineering \\ Optical Bioimaging Laboratory \\ 9 Engineering Drive 1 \\ Singapore 117576
}

\begin{abstract}
We report on a novel acousto-optic tunable filter (AOTF)based near-infrared (NIR) fluorescence excitation-emission matrix (EEM) spectroscopy technique for rapid multifluorophore characterization. We implement a unique light filtering module design by using cascaded AOTFs coupled with three orthogonally oriented polarizers to effectively remove the side-ripple artifacts of AOTFs as well as by using a pair of AOTFs coupled with two orthogonally oriented polarizers to improve detection efficiency for high-quality fluorescence EEM acquisitions. NIR fluorescence EEM spectroscopy (41 excitation wavelengths ranging from 550 to $950 \mathrm{~nm}$ in 10-nm increments; fluorescence emission from 570 to $1000 \mathrm{~nm}$ at 10-nm intervals) can be acquired from fluorescence dyes [e.g., diethylthiatricarbocyanine (DTTC) iodide, oxazine 750, and IR 140] within $10 \mathrm{~s}$ or even less, illustrating the potential of the AOTF-based NIR EEM technique developed for rapid multifluorophore analysis and characterization in biochemical and biomedical systems. ๑ 2010 Society of Photo-Optical Instrumentation Engineers. [DOI: 10.1117/1.3374051]
\end{abstract}

Keywords: acousto-optic tunable filter (AOTF); fluorescence; excitation-emission matrices (EEMs); near-infrared (NIR) fluorescence; spectroscopy.

Paper 09415R received Sep. 15, 2009; revised manuscript received Feb. 9, 2010 accepted for publication Feb. 10, 2010; published online Mar. 30, 2010.
Fluorescence spectroscopy has been studied over decades for noninvasive or minimally invasive tissue diagnosis and characterization. ${ }^{1-5}$ Fluorescence depends on exogenous or endogenous fluorophores in the tissue, which may undergo a change associated with disease transformation. ${ }^{3-5}$ The fluorophore change may be detected as an alteration in the spectral profile and intensity of fluorescence emission. ${ }^{4,5}$ Different excitation wavelengths can induce different types of tissue fluorophores to fluoresce, resulting in different spectral profiles and intensities of tissue spectra with different diagnostic abilities for disease detection. ${ }^{4}$ Fluorescence excitation-emission matrices (EEMs) spectroscopy is a powerful technique for comprehensively investigating fluorescence properties of specific fluorophores in tissues for better diagnosis and characterization. ${ }^{4,5}$ In typical existing EEM systems, four different types of tunable excitation light modules are usually used: (1) an arc lamp coupled with a monochromator or interference bandpass filters, (2) an arc lamp coupled with double monochromators, (3) a nitrogen-pumped dye laser, and/or (4) an optical parametric oscillator (OPO) tunable laser. ${ }^{3-8}$ But all these types of tunable light sources use stepper motor-type mechanisms for rotating gratings or filter

Address all correspondence to: Dr. Zhiwei Huang, National University of Singapore, Faculty of Engineering, Department of Bioengineering, Optical Bioimaging Laboratory, 9 Engineering Drive 1, Singapore 117576. Tel: 65-6516-8856; Fax: 65-6872-3069; E-mail: biehzw@nus.edu.sg wheels or crystals for tuning the excitation light wavelengths. This mechanical movement imposes limitations on the speed of tunings from one wavelength to another, resulting in lengthy EEM data acquisitions (up to minutes or hours) which are unsuitable for in vivo biomedical applications. ${ }^{4-8}$ To tackle this problem, an acousto-optic tunable filter (AOTF) can be employed to electronically tune various wavelengths of a xenon arc lamp with a high-throughput ( $>90 \%$ diffraction efficiency) within milliseconds without moving parts by varying the radio frequency $(\mathrm{RF})$ of the acoustic wave propagating through the crystal. ${ }^{9}$ To date, most EEM studies have focused on excitation wavelengths ranging between ultraviolet (UV) and shorter wavelength visible (VIS) light. ${ }^{4-7}$ Near-infrared (NIR) fluorescence EEM, which has the potential of providing the optimized excitation-emission wavelengths maxima for realizing deep-tissue imaging, has not yet been reported in the literature. In this work, we report on a novel AOTF-based NIR fluorescence excitation-emission matrix (EEM) spectroscopy technique for rapid multifluorophore analysis and characterization. The special excitation and emission AOTF filtering modules coupled with polarizers are integrated into the EEM system to effectively remove the side-ripple artifacts of AOTFs as well as to improve the collection efficiency for high-quality fluorescence EEM measurements.

$1083-3668 / 2010 / 15(2) / 027010 / 4 / \$ 25.00$ ○ 2010 SPIE 


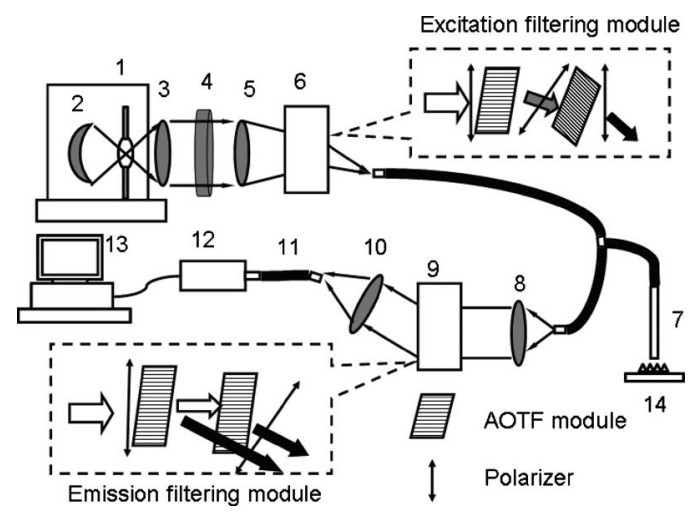

Fig. 1 Schematic diagram of the rapid near-infrared (NIR) fluorescence excitation-emission matrices (EEMs) spectroscopy system utilizing the acousto-optic tunable filter (AOTF) technique. 1 -xenon arc lamp; 2-adjustable parabolic reflector; 3, 5, 8, 10-lens; 4-hot mirror; 6-excitation filtering module; 7-bifurcate fiber-optic probe; 9-emission filtering module; 11-patch cord fiber; 12-singlephoton counting module (SPCM); 13-computer; 14-sample.

Figure 1 shows the schematic diagram of the rapid NIR fluorescence EEM spectroscopy system developed utilizing the AOTF technique. This EEM system primarily consists of a 300-W xenon arc lamp light source (emission from 300 to $1000 \mathrm{~nm}$, Newport, Inc., Stratford, Connecticut), the cascaded NIR AOTFs (MD 21152-9201, Brimrose Corp., Sparks, Maryland) coupled with three orthogonally oriented polarizers as an excitation filtering module for electronic tuning of excitation light wavelengths, a bifurcate fiber-optic probe for excitation light delivery and fluorescence light collection, a pair of AOTFs arranged in series coupled with two polarizers as an emission wavelength filtering module for fluorescence emission, and an avalanche photodiode integrated with a single-photon counting module (SPCM-AQR, PerkinElmer, Inc., Santa Clara, California) for fluorescence detection. We have also developed LabView-based software for the real-time EEM data acquisition and processing (e.g., synchronization of AOTFs for excitation and emission wavelengths tuning, SPCM dark-noise subtraction, wavelength calibration, system spectral response calibration, wavelengthdependent excitation power calibration and normalization, etc.).

To electronically continuously tune the excitation wavelengths of the xenon light source ranging from 550 to $950 \mathrm{~nm}$ with a narrow bandwidth (FWHM of $\sim 5 \mathrm{~nm}$ for each excitation wavelength) at $10-\mathrm{nm}$ increments or even smaller intervals, we devise a novel excitation filtering module into the EEM system for effective side-ripple suppression, which includes the cascaded AOTFs coupled with three polarizers (inset of Fig. 1). In this excitation filtering design, the polarization axes of the adjacent polarizers as well as of the two AOTF modules are orthogonally oriented with each other to optimize the coupling of the incident light among the different optical components. That is, the approximately collimated incident light of the xenon lamp passes through the first polarizer oriented in the perpendicular direction, and the resulting perpendicularly polarized incident light is optimally coupled into the first AOTF, such that the excitation light can be spectrally filtered out by the first AOTF along its first-order dif- fraction direction, which is well separated from the undiffractive zero-order beam. The spectrally filtered light from the first AOTF is horizontally polarized (the polarization direction of the light changes $90 \mathrm{deg}$ after the first AOTF), ${ }^{9}$ and passes through the second polarizer for further purification of the polarization status of the incident light along the horizontal direction. Then, the horizontally polarized light is coupled into the second AOTF for further spectral filtering and for another 90 -deg polarization rotation. A third polarization (oriented in the perpendicular direction) is placed after the second AOTF to block the residual horizontally polarized light while allowing the doubly spectrally filtered incident light in perpendicular polarization to come out from the excitation filtering module for fluorescence excitation. The side-ripple level of single AOTF excitation filtering module $S_{S}$ is the ratio of intensity maximum of the first side-robe band $\left(I_{S}\right)$ to the primary band transmission intensity $\left(I_{\max }\right),{ }^{9}$

$$
S_{s}=10 \log \left(\frac{I_{s}}{I_{\max }}\right)=10 \log \left(\frac{(u / 2)^{2} \cos ^{2} \delta}{\sin ^{2}(u / 2)}\right)
$$

where $u$ is the AO coupling coefficient, and $\delta$ is the smallest positive nonzero solution of equation $\tan \delta=\delta$ (Ref. 9). Without a loss of generality by assuming that the coupling coefficients $u$ of the two AOTFs are the same, the side-ripple level of the cascaded AOTFs excitation filtering module $S_{c}$ (inset of Fig. 1) can be expressed as

$$
S_{c}=10 \log \left(\frac{I_{s 1} I_{s 2}}{I_{1 \max } I_{2 \max }}\right) \approx 10 \log \left[\frac{(u / 2)^{4} \cos ^{4} \delta}{\sin ^{4}(u / 2)}\right]=2 S_{s}
$$

Hence, compared to the single AOTF filtering design, our cascaded AOTF filtering module design provides approximately two fold improvements in side-ripple suppression of the spectrally filtered excitation light. Additionally, the orthogonal polarization settings between the cascaded AOTFs and the polarizers ensure both the polarization and spatial separations of the spectrally filtered light beam (i.e., firstorder diffraction) from the nondiffraction light beam (i.e., zero order) to be double as compared to the single AOTF filtering module, thereby further reducing the out-of-band light leakage.

To identify the fluorescence emission wavelengths without mechanical scan as well as to further improve the detection of fluorescence emission from the samples, we incorporate a unique AOTF emission filtering module design into the NIR EEM technique (inset of Fig. 1). The emission filtering module comprises a pair of NIR AOTFs arranged in series (with the same incident axis and polarization orientation with each other) as well as the two orthogonally oriented polarizers. The two polarizers are placed before the first AOTF and after the second AOTF, respectively. This unique emission filtering design ensures that the total diffractive fluorescence light $I_{e m}$ from both the first and second AOTFs can pass through the second polarizer for fluorescence detection (inset of Fig. 1), which can be written as ${ }^{9}$ 


$$
I_{e m}=\sin ^{2}\left(\frac{u}{2}\right)(1-\rho)+\left[1-\sin ^{2}\left(\frac{u}{2}\right)\right] \sin ^{2}\left(\frac{u}{2}\right),
$$

where $\rho$ denotes the cross-talk coefficient between the first and second AOTFs, whereby the spectrally filtered fluorescence light from the first AOTF is collected by the second AOTF. With a proper arrangement of the separation as well as the parallel polarization orientations between the two AOTFs, the cross-talk coefficient can be minimized (i.e., $\rho \ll 1$ ). Hence, the overall fluorescence intensity $I_{e m}$ collected is rewritten as

$$
\begin{gathered}
I_{e m} \approx\left[2-\sin ^{2}\left(\frac{u}{2}\right)\right] \sin ^{2}\left(\frac{u}{2}\right)=\left(2-I_{0}\right) \cdot I_{0}, \\
I_{0}=\sin ^{2}\left(\frac{u}{2}\right),
\end{gathered}
$$

where $I_{0}(<1)$ is the maximum diffraction efficiency of a single AOTF emission filtering module. Obviously, our double AOTF emission filter module design provides a higher diffraction efficiency of fluorescence detection compared to the single AOTF filtering module.

We have verified the improvements of the out-of-band rejection and the detection efficiency for fluorescent dye measurements based on the unique excitation and emission filtering modules design in our NIR EEM technique developed. Figure 2(a) shows the comparison of transmission spectra of the excitation light at $730 \mathrm{~nm}$ from the xenon arc lamp using the cascaded AOTF-NIR EEM design and the conventional single AOTF-NIR EEM design. The out-of-band rejection in our cascaded AOTF EEM design is approximately two orders of magnitude higher than that of the single AOTF EEM design [i.e., improved from $-20 \mathrm{~dB}$ to $-40 \mathrm{~dB}$, as shown in Fig. 2(a)]. Figure 2(b) shows the comparison of fluorescence spectra of diethylthiatricarbocyanine (DTTC) iodide with a concentration of $1.84 \times 10^{-6} \mathrm{M}$ in ethanol solution acquired by using three different AOTF EEM designs: curve 1 is acquired by using our developed AOTF-NIR EEM system (Fig. 1 ); curve 2 is acquired by using the AOTF-NIR EEM system, but the excitation filtering module is replaced with a single AOTF module design; and curve 3 is obtained by using the AOTF-NIR EEM system, but the emission filtering module is replaced with a single AOTF module design. Clearly, the sideripple artifacts of the AOTF peaking at about 830, 885, 910, and $920 \mathrm{~nm}$ occur in the fluorescence spectrum measured by using the single AOTF excitation filtering module (curve 2), while these spectral contaminations are effectively removed using our cascaded AOTFs excitation filtering module designed (curves 1 and 3). The diffraction efficiency for improving fluorescence detection has also been demonstrated in the fluorescence spectrum (curve 1) observed in the NIR EEM system using the double AOTF emission filter module design (Fig. 1) as compared to curve 3 obtained in the NIR EEM system using a single AOTF emission filtering module.

We have also evaluated the repeatability and sensitivity of the developed AOTF-based NIR EEM system. Figure 3 shows the fluorescence peak intensity (at $805 \mathrm{~nm}$ ) of DTTC versus the concentration ranging from $5.74 \times 10^{-8}$ to 1.84 $\times 10^{-6} \mathrm{M}$ in ethanol solution as well as the corresponding
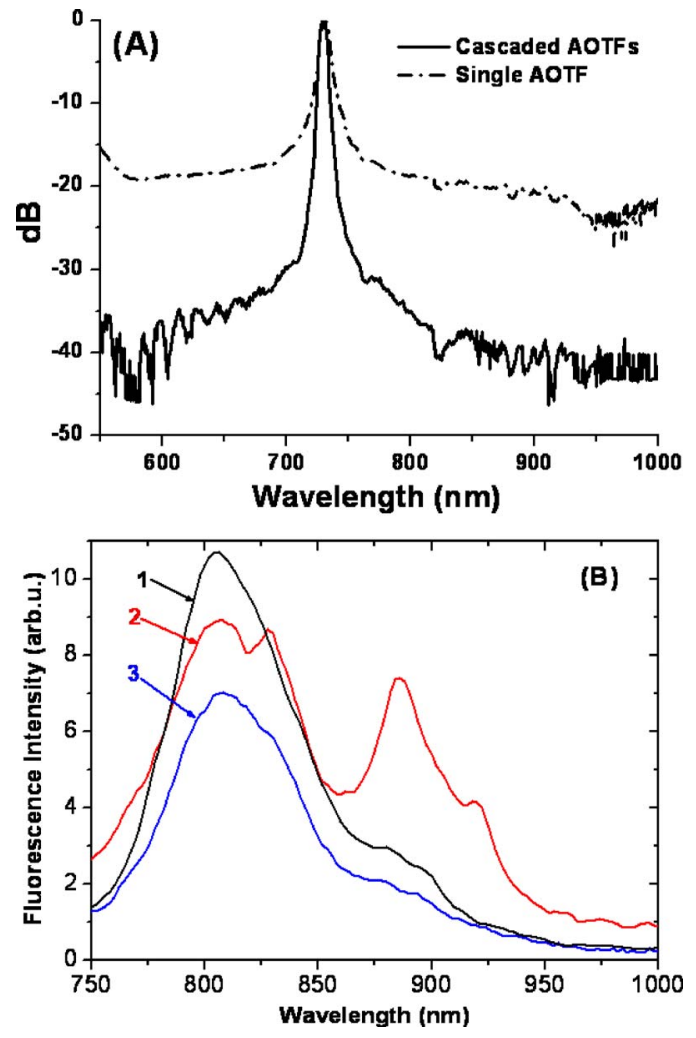

Fig. 2 (a) Comparison of transmission spectra of the excitation light at $730 \mathrm{~nm}$ from the xenon arc lamp using the cascaded AOTF-NIR EEM design and the conventional single AOTF-NIR EEM design. (b) Comparison of fluorescence spectra of diethylthiatricarbocyanine (DTTC) iodide with a concentration of $1.84 \times 10^{-6} \mathrm{M}$ in ethanol solution acquired by using three different AOTF-EEM designs under an excitation wavelength of $730 \mathrm{~nm}$. Curve 1: Fluorescence spectrum measured by the AOTF-NIR EEM system developed (Fig. 1). Curve 2: Fluorescence spectrum measured by the AOTF-NIR EEM system, but excitation filtering module is replaced by using a single AOTF module design. Curve 3: Fluorescence spectrum measured by the AOTF-NIR EEM system, but emission filtering module is replaced by using a single AOTF module design. Note that each fluorescence spectrum is normalized to its excitation power on the sample.

signal-to-noise ratios (SNRs) under the excitation wavelength of $730 \mathrm{~nm}$. The variations of fluorescence signals detected by the NIR EEM system are less than $8 \%$ (standard deviations for 10 measurements at each concentration). The fluorescence intensity observed is approximately proportional to the increased DTTC concentrations (from $5.74 \times 10^{-8}$ to 1.84 $\times 10^{-6} \mathrm{M}$ in ethanol solution), and the corresponding signalto-noise ratios (SNRs) also increase (from $2 \mathrm{~dB}$ up to $30 \mathrm{~dB}$ ) accordingly.

We also demonstrate that NIR fluorescence EEM spectroscopy (41 excitation wavelengths ranging from 550 to $950 \mathrm{~nm}$ in 10-nm increments; fluorescence emission from 570 to $1000 \mathrm{~nm}$ at $10-\mathrm{nm}$ intervals) can be acquired from multifluorophores within $10 \mathrm{~s}$ (each data point is integrated with $5 \mathrm{~ms}$ to ensure a good SNR of $>30 \mathrm{~dB}$ ) or even shorter (of a few seconds or subseconds by using a shorter integration time of $\leqslant 1 \mathrm{~ms}$ for each data point) utilizing the developed rapid AOTF-based NIR EEM technique. Figure 4 shows an example of a 2-D EEM map of the three NIR fluorescent dyes 


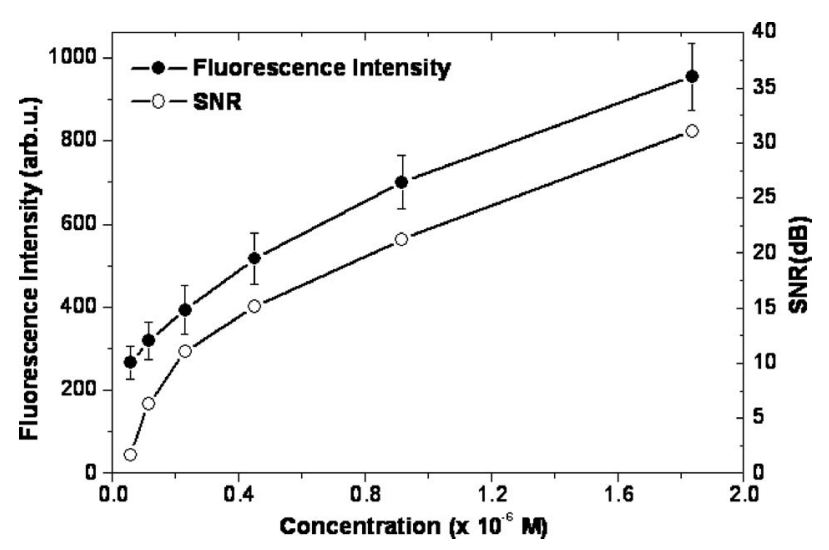

Fig. 3 Relationship of the fluorescence peak intensity (at $805 \mathrm{~nm}$ ) of DTTC versus the concentrations in ethanol solution as well as the corresponding signal-to-noise ratio (SNR) under the excitation wavelength of $730 \mathrm{~nm}$ with the incident power of $50 \mu \mathrm{W}$ on the samples. The error bars stand for the standard deviations $(<8 \%)$ of the 10 measurements of DTTC solutions for each concentration.

(oxazine 750 at $2.13 \times 10^{-6} \mathrm{M}$, DTTC iodide at 1.84 $\times 10^{-6} \mathrm{M}$, and IR 140 dye at $1.28 \times 10^{-6} \mathrm{M}$ ) mixed in ethanol solution that is acquired in $10 \mathrm{~s}$ with an SNR of over $20 \mathrm{~dB}$. The excitation-emission maxima of the three dyes-Ex $=670 \mathrm{~nm}: \quad \mathrm{Em}=695 \mathrm{~nm}$ for oxazine750; $\mathrm{Ex}$ $=780 \mathrm{~nm}$ : Em=800 nm for DTTC iodide; and Ex=810 nm: $\mathrm{Em}=850 \mathrm{~nm}$ for IR 140 - are clearly identified using our AOTF-based NIR EEM technique. The slight shifts of the EEM maxima of each fluorescent dye observed in mixtures as compared to the literature report ${ }^{10}$ are probably due to the intersystem energy transfers among the different fluorescent dyes in ethanol solution. ${ }^{11}$ As such, our rapid AOTF-NIR EEM technique may also be useful for monitoring the relative stability of the fluorescence dyes as well as the extent of energy transfer between different fluorescent dyes in mixtures followed by different light irradiations.

In conclusion, a novel, AOTF-based NIR EEM spectroscopy system for rapid analysis of multifluorophores has been designed, constructed, and evaluated. This NIR EEM system

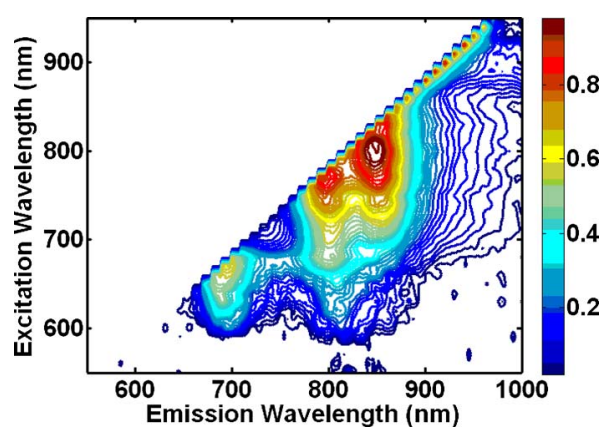

Fig. 4 2-D EEM map of the three fluorescent dyes mixtures (oxazine 750 at $2.13 \times 10^{-6} \mathrm{M}$, DTTC iodide at $1.84 \times 10^{-6} \mathrm{M}$, and IR 140 dye at $1.28 \times 10^{-6} \mathrm{M}$ mixed in ethanol solution) acquired in $30 \mathrm{~s}$ under excitation wavelengths ranging from 550 to $950 \mathrm{~nm}$ at a $10-\mathrm{nm}$ increment, and emission wavelengths ranging from 570 to $1000 \mathrm{~nm}$ at 10-nm intervals. constitutes the advantages of fully electronic tuning abilities of wavelengths without any mechanical scanning parts, thereby enabling fast excitation and emission wavelength tuning, large sizes of excitation-emission data matrices for multifluorophore analysis, as well as high reproducibility of EEM scans in a rapid manner. The unique excitation and emission filtering modules design ensures an effective suppression of side-ripple artifacts of AOTFs as well as improves the detection efficiency for high-quality fluorescence NIR EEM measurements. The NIR EEM technique developed with short measurement times will make in vivo measurements convenient and efficient, which has the potential to move this strict research tool into clinical research and practice in clinical settings. One notes that although our current EEM system is designed for long-wavelength VIS/NIR bands, where currently available AOTFs have optimum transmission efficiency, it can also be readily modified into an EEM system for UV/VIS bands by replacing the VIS/NIR AOTFs with highperformance UV/VIS AOTFs (e.g., quartz or potassium dihydrogen phosphate (KDP) crystal-based AOTFs). ${ }^{12,13}$

\section{Acknowledgments}

This research is supported by the Biomedical Research Council, the National Medical Research Council, and the Faculty Research Fund from the National University of Singapore.

\section{References}

1. J. Hung, S. Lam, J. C. LeRiche, and B. Palcic, "Autofluorescence of normal and malignant bronchial tissue," Lasers Surg. Med. 11, 99105 (1991).

2. R. R. Alfano, G. C. Tang, A. Pradham, W. Lam, D. S. J. Choy, and E. Opher, "Fluorescence spectra from cancerous and normal human breast and lung tissues," IEEE J. Quantum Electron. QE23, 18061811 (1987).

3. Z. Huang, W. Zheng, S. Xie, R. Chen, H. Zeng, D. I. McLean, and H. Lui, "Laser-induced autofluorescence microscopy of normal and tumor human colonic tissue," Int. J. Oncol. 24, 59-63 (2004).

4. R. Richards-Kortum, R. P. Rava, R. E. Petras, M. Fitzmaurice, M. Sivak, and M. S. Feld, "Spectroscopic diagnosis of colonic dysplasia," Photochem. Photobiol. 53, 777-786 (1991).

5. W. Zheng, W. Lau, C. Cheng, K. C. Soo, and M. Olivo, "Optimal excitation-emission wavelength for autofluorescence diagnosis of bladder tumors," Int. J. Cancer 104, 477-481 (2003).

6. C. MacAulay, R. Richards-Kortum, U.Utzinger, A. Fedyk, E. N. Atkinson, D. Cox, and M. Follen, "Variation of fluorescence spectroscopy during the menstrual cycle," Opt. Express 10, 493-504 (2002).

7. R. S. DaCosta, H. Andersson, and B. C. Wilson, "Molecular fluorescence excitation-emission matrices relevant to tissue spectroscopy," Photochem. Photobiol. 78, 384-392 (2003).

8. M. Gouzman, N. Lifshitz, S. Luryi, O. Semyonov, D. Gavrilov, and V. Kuzminskiy, "Excitation-emission fluorimeter based on linear interference filters," Appl. Opt. 43, 3066-3071 (2004).

9. I. C. Chang, "Acousto-optic devices and applications," IEEE Trans. Sonics Ultrason. Sup 23, 2-22 (1976).

10. B. M. Pierce and R. R. Birge, "Lasing properties of several near-IR dyes for a nitrogen laser-pumped dye laser with an optical amplifier," IEEE J. Quantum Electron. QE18, 1164-1170 (1982).

11. C. Clendinen, C. Clendinen, W. N. Sisk, H. Nishikiori, N. Tanaka, and T. Fuji, "Energy transfer and photodegradation of perylene red with DTTC and HITC acceptor dyes in PMMA," Dyes Pigm. 77, 92-97 (2008).

12. N. S. Prasad, "Deep-UV based acousto-optic tunable filter for spectral sensing applications," Int. J. High Speed Electron. Syst. 17, 857866 (2007).

13. V. Voloshinov and N. Gupta, "Ultraviolet-visible imaging acoustooptic tunable filters in KDP," Appl. Opt. 43, 3901-3909 (2004). 